\title{
SPARSE ADAPTIVE MULTIPATH TRACKING FOR LOW BANDWIDTH RANGING APPLICATIONS
}

\author{
Nicolas Schneckenburger and Dmitriy Shutin \\ German Aerospace Center (DLR) \\ Institute of Communication and Navigation \\ Oberpfaffenhofen, Germany
}

\begin{abstract}
In this paper a novel algorithm for estimation and tracking of multipath components for range estimation using signals with low bandwidth is discussed. In multipath rich environments ranging becomes a challenging problem when used with low bandwidth signals: unless multipath interference is resolved, large ranging errors are typical. In this work the estimation and tracking of individual multipath components is studied. The new technique combines sparse Bayesian learning and variational Bayesian parameter estimation with Kalman filtering. While the former is used to detect and estimate the individual components, the Kalman filtering is used to track the estimated signals. Two assumptions are compared: independence of multipath components, typical for classical multipath estimation schemes, versus correlation between the propagation paths. The later has been found to improve component tracking and estimation at the cost of increased computational complexity. The performance of the algorithm is investigated using synthetic, as well as real measurement data collected during flight trials. Significantly improved ranging performance can be obtained as compared to the standard correlation-based ranging.
\end{abstract}

\section{INTRODUCTION}

The goal of this paper is the estimation and tracking of the line of sight (LOS) path between the transmitter and receiver for ranging applications in multipath environments. This problem is well known in navigation systems, such as GPS, that rely on the estimation of a range (or pseudorange) for position calculation. Practically, the received signal often consists of a superposition of several copies of the transmitted signal arriving at the receiver via different propagation paths - multipath components. This leads to a decrease of the ranging accuracy. A possible solution to this problem is trying to resolve the individual propagation paths from the superimposed signal to reduce the interference. However, the ability to separate the different paths strongly depends on the bandwidth of the transmitted signal. For GPS with a bandwidth of $10.23 \mathrm{MHz}$, multipaths may be separated up to a few meters.

However, when bandwidth is low, the multipath problem becomes a challenge. Specifically, a new L-band aeronautical communication system type 1 (LDACS1) [1] has been recently proposed to implement a navigational functionality and serve as a backup for GPS in civil aeronautics. Due to a lack of spectrum, the system allocates the channels in spectral holes between other systems in L-band. The used bandwidth of $500 \mathrm{kHz}$ allows separating multipath that are several hundreds of meters apart, yet for ground-based systems, separations up to tens of meters are not uncommon. Specifically, measurement results indicate that standard correlator processing leads to ranging errors up to several $100 \mathrm{~m}[2,3]$. Thus, algorithms that able to perform robust ranging in multipath environments are required.

Let us consider the following discrete time model of a multipath channel (see also, e.g., [4]): The received signal, due to the multipath propagation can be represented as a superposition of $L$ specular propagation paths

$$
\boldsymbol{y}=\sum_{l=1}^{L} \boldsymbol{s}\left(\boldsymbol{\theta}_{l}\right) w_{l}+\boldsymbol{\xi}=\boldsymbol{S}(\boldsymbol{\Theta}) \boldsymbol{w}+\boldsymbol{\xi},
$$

where each path consists of a transmitted signal $s$, nonlinearly parameterized by $\boldsymbol{\theta}_{l}$. In our case, the nonlinear parametrization $\boldsymbol{\theta}_{l}$ simply includes a delay of the original transmitted signal $s(t)$ by $\tau_{l}$, and a possible Doppler shift $\nu_{l}$. The weights $w_{l}$ represent the attenuation and a constant phase shift of each path. Finally, the random perturbation $\boldsymbol{\xi}$ represents the measurement noise. It is assumed to be normally distributed with zero mean and covariance $\boldsymbol{\Sigma}=\lambda^{-1} \boldsymbol{I}$. The LOS path, if present, is the path with the shortest delay $\tau_{l}$. Adding to that, usually several different non direct paths, the non line of sight (NLOS) paths exist. Our goal is to estimate and track the LOS over time using the measured signal $\boldsymbol{y}$. For simplification, we split this problem into two parts: first, we estimate the number of signals in (1) and their parameters $\boldsymbol{\Theta}=\left[\boldsymbol{\theta}_{1}, \ldots, \boldsymbol{\theta}_{L}\right]$; then, the estimated components are tracked over time and the LOS is determined.

The estimation of signal parameters $\Theta$ and $\boldsymbol{w}$ has often been solved using Expectation-Maximization (EM) type of algorithms [57]. Due to the nonlinearity of (1) with respect to the parameter set $\Theta$, such algorithms significantly simplify the numerical optimization, yet they are applicable only when the order $L$ of the model is known and fixed - an assumption that is rarely satisfied in practice. However, model order can be assessed by introducing sparsity constraints into the estimation, thus allowing joint model order selection and parameter estimation. With a few minor variations, the general goal of sparse reconstruction is to optimally estimate the parameters $\boldsymbol{w}$ of the model (1) with fixed design matrix $\boldsymbol{S}(\boldsymbol{\Theta}) \equiv \widetilde{\boldsymbol{S}}$. The sparse solution is obtained by imposing specific sparsity constraints on the signal parameter $\boldsymbol{w}[8,9]$. Here we will make use of a special class of sparsity techniques, known as Sparse Bayesian learning (SBL) $[4,10,11]$. SBL is a family of empirical Bayes techniques that finds a sparse estimate of $\boldsymbol{w}$ by modeling the weights using a hierarchical prior $p(\boldsymbol{w} \mid \boldsymbol{\alpha}) p(\boldsymbol{\alpha})=\prod_{l=1}^{L} p\left(w_{l} \mid \alpha_{l}\right) p\left(\alpha_{l}\right)$, where $p\left(w_{l} \mid \alpha_{l}\right)$ is a Gaussian probability density function (pdf) with zero mean and precision parameter $\alpha_{l}$, also called the sparsity parameter; larger values of $\alpha_{l}$ drive the corresponding weight toward zero, thus encouraging a sparse solution. One particular method for SBL recently proposed in the literature is a fast variational SBL (FV-SBL) [4]. The FV-SBL algorithm optimizes the corresponding objective func- 
tion - the variational lower bound on the model evidence $\log p(\boldsymbol{y})$ - incrementally, i.e., with respect to one basis function at a time. Such an optimization strategy is in many respects similar to the incremental estimation of the multipath parameters employed in the classical SAGE algorithm [7] and its variational Bayesian extension - VB-SAGE algorithm [12].

For the tracking of multipath components, different approaches have been considered. In [13] the different components of a MIMO channel were estimated using a generalized EM algorithm and tracked using a Kalman filter. The model order is determined using minimum description length (MDL) criteria [14]. As the algorithm is developed for channel sounding, no decision on line of sight is made. Also, the algorithm is very computationally expensive as MDL reuqires training multiple models in parallel. In $[15,16]$ a particle filter is employed for the tracking of the LOS component in satellite navigation. However, due to the very nature of a particle filters they are computational expensive and in general lack the super-resolution property.

In this paper we combine sparsity-based joint model order selection and parameter estimation with Kalman-based component tracking for ranging applications. For the super-resolution calculation of the parameters SBL technique is applied and builds on two key concepts: variational Bayesian estimation of signal parameters $\Theta$, and an incremental FV-SBL algorithm [4]. In contrast to the VB-SAGE algorithm [17], where multipath components are assumed to be independent, the FV-SBL based estimation of signal parameters considers correlations between components [18]. The impact of these correlations on the model order selection and tracking of LOS component is analyzed.

Throughout the paper we make use of the following notation. Vectors and matrices are represented as, respectively, boldface lowercase letters, e.g., $\boldsymbol{x}$, and boldface uppercase letters, e.g., $\boldsymbol{X}$. We use $\boldsymbol{e}_{l}=\left[0_{1}, \ldots, 0_{l-1}, 1_{l}, 0_{l+1}, \ldots, 0_{L}\right]^{T}$ to denote a canonical vector of appropriate dimension. Finally, for a random vector $\boldsymbol{x}$, $\mathrm{CN}(\boldsymbol{x} \mid \boldsymbol{a}, \boldsymbol{B})$ denotes a circular symmetric normal distribution pdf with mean $\boldsymbol{a}$ and covariance matrix $\boldsymbol{B}$; similarly, for a random variable $x, \mathrm{Ga}(x \mid a, b)=\frac{b^{a}}{\Gamma(a)} x^{a-1} \exp (-b x)$ denotes a gamma pdf with parameters $a$ and $b$.

\section{SIGNAL MODEL AND ADAPTIVE FAST SPARSE BAYESIAN LEARNING}

In the following the variational Bayesian parameter estimation algorithms are described. For more details the reader is referred to the original publications [4] and [18].

The joint pdf variables from (1) and the sparsity parameters can be represented as $p(\boldsymbol{w}, \lambda, \boldsymbol{\alpha}, \boldsymbol{\Theta}, \boldsymbol{y})=p(\boldsymbol{y} \mid \boldsymbol{w}, \lambda, \boldsymbol{\Theta}) p(\boldsymbol{w} \mid \boldsymbol{\alpha}) p(\boldsymbol{\alpha})$ $p(\lambda) p(\boldsymbol{\Theta})$, where $p(\boldsymbol{y} \mid \boldsymbol{w}, \lambda, \boldsymbol{\Theta})=\operatorname{CN}\left(\boldsymbol{y} \mid \boldsymbol{S}(\boldsymbol{\Theta}) \boldsymbol{w}, \lambda^{-1} \boldsymbol{I}\right), p(\boldsymbol{w} \mid \boldsymbol{\alpha})=$ $\prod_{l=1}^{L} \mathrm{CN}\left(w_{l} \mid 0, \alpha_{l}^{-1}\right), p(\boldsymbol{\alpha}) \propto \prod_{l=1}^{L} \alpha_{l}^{-1}$, and $p(\lambda) \propto \lambda^{-1}$, following the standard SBL model assumption $[4,19]$. The choice of the prior $p(\boldsymbol{\Theta})=\prod_{l=1}^{L} p\left(\tau_{l}\right) p\left(\nu_{l}\right)$ is arbitrary in the context of this work and is generally application specific. The variational inference on this graph aims at estimating a "proxy" pdf $q(\boldsymbol{w}, \boldsymbol{\alpha}, \lambda, \boldsymbol{\Theta})$ that maximizes the lower bound on the $\log$-evidence $\log p(\boldsymbol{y})$ [20]:

$$
\log p(\boldsymbol{y}) \geq \underset{q(\boldsymbol{w}, \boldsymbol{\alpha}, \lambda, \boldsymbol{\Theta})}{\mathbb{E}} \log \frac{p(\boldsymbol{w}, \lambda, \boldsymbol{\alpha}, \boldsymbol{\Theta}, \boldsymbol{y})}{q(\boldsymbol{w}, \boldsymbol{\alpha}, \lambda, \boldsymbol{\Theta})}
$$

For the factorization of $q(\boldsymbol{w}, \boldsymbol{\alpha}, \lambda, \boldsymbol{\Theta})$ two different assumptions can be made: If the individual components are assumed to be indepen- dent, $q(\boldsymbol{w}, \boldsymbol{\alpha}, \lambda, \boldsymbol{\Theta})$ can be expressed as

$$
q_{\mathrm{U}}(\boldsymbol{w}, \boldsymbol{\alpha}, \lambda, \boldsymbol{\Theta})=q(\lambda) \prod_{l=1}^{L} q\left(\alpha_{l}\right) q\left(\boldsymbol{\theta}_{l}\right) q\left(w_{l}\right),
$$

This assumption is made in the VB-SAGE algorithm [12], as well as (implicitly) in the SAGE algorithm [7]. However, it is also possible to assume a correlation between the gains of the different components, as it is done in the classical SBL scheme. In this case, $q_{\mathrm{C}}(\boldsymbol{w}, \boldsymbol{\alpha}, \lambda, \boldsymbol{\Theta})$ factors as

$$
q_{\mathrm{C}}(\boldsymbol{w}, \boldsymbol{\alpha}, \lambda, \boldsymbol{\Theta})=q(\boldsymbol{w}) q(\lambda) \prod_{l=1}^{L} q\left(\alpha_{l}\right) q\left(\boldsymbol{\theta}_{l}\right) .
$$

The variational factors in (3) and (4) are chosen as: $q(\boldsymbol{w})=$ $\mathrm{CN}(\boldsymbol{w} \mid \widehat{\boldsymbol{w}}, \widehat{\boldsymbol{\Phi}}), q\left(\alpha_{l}\right)=\mathrm{Ga}\left(\alpha_{l} \mid 1, \widehat{\alpha}_{l}^{-1}\right)$, and $q(\lambda)=\mathrm{Ga}\left(\lambda \mid N / 2, N \widehat{\lambda}^{-1} / 2\right)$. In case of parameters $\boldsymbol{\Theta}$ we assume that $q\left(\boldsymbol{\theta}_{l}\right)=\delta\left(\boldsymbol{\theta}_{l}-\widehat{\boldsymbol{\theta}}_{l}\right)$, i.e. we calculate point estimates rather than distributions of these parameters ${ }^{1}$. The optimal $q(\boldsymbol{w}, \boldsymbol{\alpha}, \lambda, \boldsymbol{\Theta})$ is then found by maximizing (2) with respect to the parameters $\left\{\widehat{\boldsymbol{w}}, \widehat{\boldsymbol{\Phi}}, \widehat{\lambda}, \widehat{\alpha}_{1}, \widehat{\boldsymbol{\theta}}_{1}, \ldots, \widehat{\alpha}_{L}, \widehat{\boldsymbol{\theta}}_{l}\right\}$ by cycling through all factors in a "round-robin" fashion [20]. The parameters of the pdf $q(\boldsymbol{w})$ can be computed as

$$
\begin{gathered}
\widehat{\boldsymbol{\Phi}}_{U}=\left(\widehat{\lambda} \boldsymbol{S}(\widehat{\boldsymbol{\Theta}})^{H} \boldsymbol{S}(\widehat{\boldsymbol{\Theta}})+\operatorname{diag}(\widehat{\boldsymbol{\alpha}})\right)^{-1}, \\
\widehat{\boldsymbol{\Phi}}_{C}=\left(\widehat{\lambda} \operatorname{diag}\left(\boldsymbol{S}(\widehat{\boldsymbol{\Theta}})^{H} \boldsymbol{S}(\widehat{\boldsymbol{\Theta}})\right)+\operatorname{diag}(\widehat{\boldsymbol{\alpha}})\right)^{-1} \\
\widehat{\boldsymbol{w}}=\widehat{\lambda} \widehat{\boldsymbol{\Phi}}_{(\cdot)} \boldsymbol{S}(\widehat{\boldsymbol{\Theta}})^{H} \boldsymbol{y},
\end{gathered}
$$

where $\widehat{\boldsymbol{\Phi}}_{(\cdot)}=\widehat{\boldsymbol{\Phi}}_{U}$ if model (3) is used and $\widehat{\boldsymbol{\Phi}}_{(\cdot)}=\widehat{\boldsymbol{\Phi}}_{C}$ in case of (4). The variational update expression for the parameter of $q(\lambda)$ is given as

$$
\widehat{\lambda}=N /\left(\|\boldsymbol{t}-\widehat{\boldsymbol{S}} \widehat{\boldsymbol{w}}\|^{2}+\operatorname{Trace}\left(\widehat{\mathbf{\Phi}} \widehat{\boldsymbol{S}}^{H} \widehat{\boldsymbol{S}}\right)\right) .
$$

In case of $q\left(\alpha_{l}\right)$, the standard variational inference of its parameters can be significantly accelerated, if the variational updates of $q\left(w_{l}\right)$ and $q\left(\alpha_{l}\right)$ (in case of model (4)), or $q(\boldsymbol{w})$ and $q\left(\alpha_{l}\right)$ (in case of model (3)) are computed for a single fixed component $l$ repeatedly ad infinitum. The corresponding stationary points of $q\left(\alpha_{l}\right)$ can then be computed analytically $[4,7]$. To this end we compute

$$
\begin{gathered}
\overline{\boldsymbol{\Phi}}_{U}=\left(\widehat{\lambda} \boldsymbol{S}(\widehat{\boldsymbol{\Theta}})^{H} \boldsymbol{S}(\widehat{\boldsymbol{\Theta}})+\sum_{k \neq l} \widehat{\alpha} \boldsymbol{e}_{k} \boldsymbol{e}_{k}^{T}\right)^{-1} \\
\overline{\boldsymbol{\Phi}}_{C}=\left(\widehat{\lambda} \operatorname{diag}\left(\boldsymbol{S}(\widehat{\boldsymbol{\Theta}})^{H} \boldsymbol{S}(\widehat{\boldsymbol{\Theta}})\right)+\sum_{k \neq l} \widehat{\alpha} \boldsymbol{e}_{k} \boldsymbol{e}_{k}^{T}\right)^{-1}, \\
\varsigma_{l}=\boldsymbol{e}_{l}^{T} \overline{\boldsymbol{\Phi}}_{(\cdot)} \boldsymbol{e}_{l} \quad \text { and } \omega_{l}^{2}=\left|\widehat{\lambda} \boldsymbol{e}_{l}^{T} \overline{\boldsymbol{\Phi}}_{(\cdot)} \boldsymbol{S}(\widehat{\boldsymbol{\Theta}})^{H} \boldsymbol{y}\right|^{2},
\end{gathered}
$$

where as before, $\overline{\mathbf{\Phi}}_{(\cdot)}=\overline{\mathbf{\Phi}}_{U}$ if model (3) is used, and $\overline{\boldsymbol{\Phi}}_{(\cdot)}=\overline{\mathbf{\Phi}}_{C}$ in case of (4). Then, the variational parameter $\widehat{\alpha}_{l}$ of $q\left(\alpha_{l}\right)$ can be computed as

$$
\widehat{\alpha}_{l}= \begin{cases}\left(\omega_{l}^{2}-\varsigma_{l}\right)^{-1}, & \omega_{l}^{2}>\varsigma_{l} \\ \infty, & \omega_{l}^{2} \leq \varsigma_{l} .\end{cases}
$$

\footnotetext{
${ }^{1}$ As a point estimate we understand maximum likelihood or maximum $a$ posteriori estimation; the latter case is automatically obtained when a prior $p\left(\boldsymbol{\theta}_{l}\right) \neq$ const.
} 
Expression (12) allows one to assess the impact of the $l$ th component on the variational lower bound: a finite value of $\widehat{\alpha}_{l}$ instructs us to keep the $l$ th component since it increases the lower bound an infinite value of $\widehat{\alpha}_{l}$ indicates that the component should be removed. In this way the model order is estimated within the estimation scheme. To compute $q\left(\boldsymbol{\theta}_{l}\right)$ the standard variational inference is used [20]. This bound is maximized when the Kullback-Leibler divergence between $q\left(\boldsymbol{\theta}_{l}\right)$ and $\tilde{p}\left(\boldsymbol{\theta}_{l}\right)$ is minimal. Note that since $q\left(\boldsymbol{\theta}_{l}\right)$ is constrained to be a Dirac distribution, the variational bound with respect to $q\left(\boldsymbol{\theta}_{l}\right)$ is maximized when $q\left(\boldsymbol{\theta}_{l}\right)$ is aligned with the mode of $\tilde{p}\left(\boldsymbol{\theta}_{l}\right) \propto \exp \left(\mathbb{E}_{q\left(\boldsymbol{w}, \lambda, \boldsymbol{\Theta}_{\bar{l}}\right)} \log p(\boldsymbol{y} \mid \boldsymbol{w}, \boldsymbol{\Theta}, \lambda) p(\boldsymbol{\Theta})\right)$. By evaluating $\tilde{p}\left(\boldsymbol{\theta}_{l}\right)$ we find the estimates for the delay $\tau_{l}$ as

$$
\begin{aligned}
& \widehat{\tau}_{l}=\underset{\tau_{l}}{\operatorname{argmax}}\left\{\log p\left(\tau_{l}, \hat{\nu}_{l}\right)-\widehat{\lambda}\left\|\boldsymbol{r}_{l}-\widehat{w}_{l} \boldsymbol{s}\left(\tau_{l}, \hat{\nu}_{l}\right)\right\|^{2}\right. \\
& \left.-\widehat{\lambda} \sum_{k \neq l} 2 \Re\left\{\Phi_{k l} \boldsymbol{s}\left(\widehat{\boldsymbol{\tau}}_{k}\right)^{H} \boldsymbol{s}\left(\tau_{l}, \hat{\nu}_{l}\right)\right\}-\widehat{\lambda} \Phi_{l l}\left\|\boldsymbol{s}\left(\tau_{l}, \hat{\nu}_{l}\right)\right\|^{2}\right\},
\end{aligned}
$$

and for the Doppler frequency $\nu_{l}$ as

$$
\begin{aligned}
& \widehat{\nu}_{l}=\underset{\nu_{l}}{\operatorname{argmax}}\left\{\log p\left(\nu_{l}, \hat{\tau}_{l}\right)-\widehat{\lambda}\left\|\boldsymbol{r}_{l}-\widehat{w}_{l} \boldsymbol{s}\left(\nu_{l}, \hat{\tau}_{l}\right)\right\|^{2}\right. \\
& \left.-\widehat{\lambda} \sum_{k \neq l} 2 \Re\left\{\Phi_{k l} \boldsymbol{s}\left(\widehat{\boldsymbol{\nu}}_{k}\right)^{H} \boldsymbol{s}\left(\nu_{l}, \hat{\tau}_{l}\right)\right\}-\widehat{\lambda} \Phi_{l l}\left\|\boldsymbol{s}\left(\nu_{l}, \hat{\tau}_{l}\right)\right\|^{2}\right\},
\end{aligned}
$$

with the residuum $\boldsymbol{r}_{l}$ being defined as

$$
\boldsymbol{r}_{l}=\boldsymbol{y}-\sum_{k=1, k \neq l}^{L} \widehat{w}_{k} \boldsymbol{s}\left(\widehat{\boldsymbol{\theta}}_{k}\right) .
$$

Let us stress that these above expression under assumption (3) lead to a version of the VB-SAGE algorithm.

\section{SPARSE ADAPTIVE MULTIPATH ESTIMATION}

The two algorithms from the previous chapter are however not adaptive, but snapshot based. To make the schemes adapt to time varying scenarios, we employ Kalman filters [21], resulting in the sparse adaptive multipath estimation algorithm (SAME). Hereby, the main challenge is to allocate the output of the super-resolution algorithm to the paths currently tracked in the Kalman filter. This decision is made comparing their estimated parameters such is amplitude, delay and Doppler shift. Assume the we are currently tracking $M$ paths within the Kalman filter and the super-resolution algorithm detects $L$ components. If $M=L$, each detected path is associated with the one showing the largest resemblance of its parameters. If $M<L$, a new path is created within the Kalman filter. The $M>L$ case means, that we are currently tracking a path that no longer exists. Hence, if a path has not been updated within a certain time, it is deleted.

For each time instance $k$, the super resolution algorithm returns a set of estimated weights $\widehat{\boldsymbol{w}}[k]$ and path parameters $\widehat{\boldsymbol{\Theta}}[k]$, in our case delay $\tau_{l}$ and Doppler frequency $\nu_{l}$, for each detected propagation path. For simplification, we denote the weights and parameters to be tracked as $\hat{\boldsymbol{\Omega}}[k]=\left[\widehat{\boldsymbol{w}}^{T}[k], \widehat{\boldsymbol{\Theta}}^{T}[k]\right]^{T}$. For all $\boldsymbol{\vartheta}_{l}[k] \in \boldsymbol{\Omega}[k]$, i.e. the weight and entire set of parameters of path $l$ at the time instance $k$, the following first order vector random walk model is assumed:

$$
\boldsymbol{\vartheta}_{l}[k]=\boldsymbol{\vartheta}_{l}[k-1]+\boldsymbol{\xi}_{e}[k], \quad N\left(\boldsymbol{\xi}_{e} ; \mathbf{0}, \boldsymbol{\sigma}_{e}^{2} \cdot \boldsymbol{I}\right) .
$$

The excitation noise $\boldsymbol{\xi}_{e}$ drives the change of each parameter over time. The distributions for each parameter are chosen according to statistics acquired during the measurement campaign. Parameters of an individual component, i.e. the weight $w_{l}$, the delay $\tau_{l}$, and Doppler frequency $\nu_{l}$ are assumed to be uncorrelated.

All estimates $\boldsymbol{\vartheta}_{l}$ of the super resolution algorithm are based on the measured data, and thus contain the measurement noise $\boldsymbol{\xi}_{m}[k]$

$$
\widehat{\boldsymbol{\vartheta}}_{l}[k]=\boldsymbol{\vartheta}_{l}[k]+\boldsymbol{\xi}_{m}[k], \quad N\left(\boldsymbol{\xi}_{m} ; \mathbf{0}, \boldsymbol{\sigma}_{m}^{2}[k] \cdot \boldsymbol{I}\right) .
$$

The noise is assumed Gaussian and its variance is estimated using both the signal to noise ratio for the entire receive signal and the amplitude estimated by the super-resolution algorithm. Using that information, an individual signal to noise ratio for every component is calculated. Based on that, the measurement variance $\boldsymbol{\sigma}_{m}^{2}$ is estimated.

Using the Kalman filter equations [21], for each time step $k$ and component $l$, estimates for the parameters $\widehat{\boldsymbol{\mu}}_{\vartheta, l}[k]$ as well as their variance $\widehat{\boldsymbol{\sigma}}_{\boldsymbol{\vartheta}, l}^{2}[k]$ are calculated, i.e. the Kalman filter models distribution of the estimated parameters for path $l$ as Gaussian pdf $\mathrm{N}\left(\widehat{\boldsymbol{p}}_{l}(\boldsymbol{\vartheta}) \mid \widehat{\boldsymbol{\mu}}_{\vartheta_{\ell} l}[k], \widehat{\boldsymbol{\sigma}}_{\boldsymbol{\vartheta}, l}^{2}[k]\right)$. Under the assumption of independent parameters, $\widehat{\boldsymbol{p}}_{l}(\boldsymbol{\vartheta})$ factors as $\widehat{\boldsymbol{p}}_{l}(\boldsymbol{\vartheta})=p\left(w_{l}\right) p\left(\tau_{l}\right) p\left(\nu_{l}\right)$. This modeling provides a convenient metric for the association of the components detected by the super resolution algorithm to the propagation paths tracked in the Kalman filter.

Assume $M$ different components are being tracked in the filter and thus providing $M$ Gaussian pdfs $\widehat{\boldsymbol{p}}_{m}(\boldsymbol{\vartheta})$. The logarithmic metric $d_{i m}$ between each of the $I$ components with parameters $\boldsymbol{\vartheta}_{l}[k]$, detected by the super-resolution algorithm, belonging to one the $M$ tracked paths can simply be evaluated by inserting each parameter vector in the pdf defined in $\widehat{\boldsymbol{p}}_{m}(\boldsymbol{\vartheta})$. Due to the factorization of $\widehat{\boldsymbol{p}}_{l}(\boldsymbol{\vartheta})$, the resulting $d_{l m}$ can be written as $d_{l m}=\log p\left(w_{l}\right)+$ $\log p\left(\tau_{l}\right)+\log p\left(\nu_{l}\right)$. Using $d_{l m}, I$ detected components can be allocated to $M$ tracked paths in the filter using the following association function: $A\left(\boldsymbol{\theta}_{l}\right)=\operatorname{argmax}_{m} d_{l m}$. If no matching path with a metric under a certain threshold is tracked, i.e. $\operatorname{argmax}_{m} d_{l m}<\psi_{\text {min }}$, a new path is generated. To avoid tracking of no longer existing paths in the filter, it is removed, if it has not been updated within a certain time $\psi_{\text {Lifetime }}$.

It is important to stress, that using this scheme, also the superresolution can benefit from the Kalman filter: Hereby, each Kalman prediction is passed as initialization to the parameter estimation algorithm. This significantly reduces the occurrence artifacts often experienced if no initialization is used.

\section{RESULTS}

In this section, we show the results of the two different algorithms described above. We start with synthetic data and then continue to real world measurement data, acquired during flight trials carried out in November 2012 [2,3].

The transmit signal is generated according to the LDACS1 standard, currently one of the most promising candidates for the future air-traffic management (ATM) data link [1]. LDACS1 uses OFDM as modulation, accommodates has 50 subcarriers into a $500 \mathrm{kHz}$ bandwidth and is planned to be employed in the aeronautical Lband. Each OFDM symbol, consisting of a useful symbol duration of $102.4 \mu$ s, is extended into a cyclic prefix (CP) of length $4.8 \mu$ s and an additional windowing time of $12.8 \mu$ s in order to reduce out of band radiation. Assuming the speed of light in air, one sample equals the distance of about $480 \mathrm{~m}$. The largest entity of the LDACS1 signal is a super-frame of length $240 \mathrm{~ms}$. For the synthetic data it is assumed to be continuously transmitted. Due to restrictions of the setup, for 


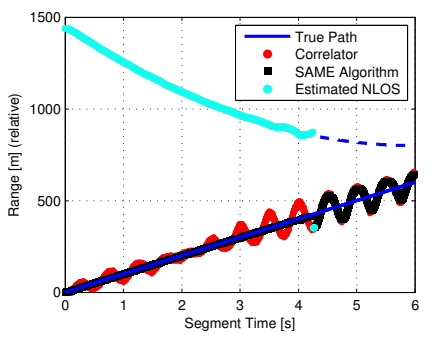

(a) Independent components

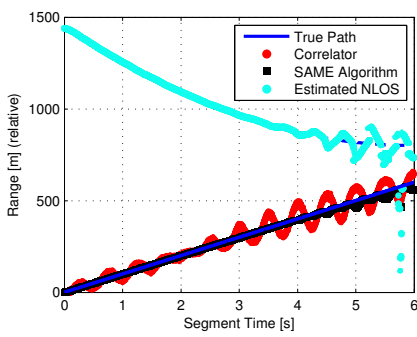

(b) Correlated components
Fig. 1: Estimated LOS and NLOS components for simulated data.

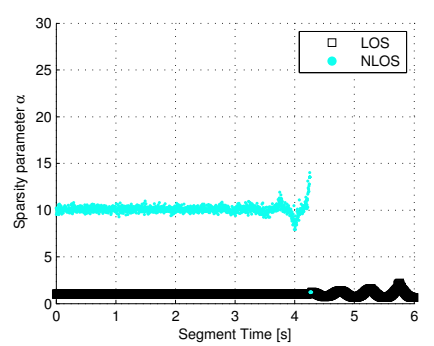

(a) Independent components

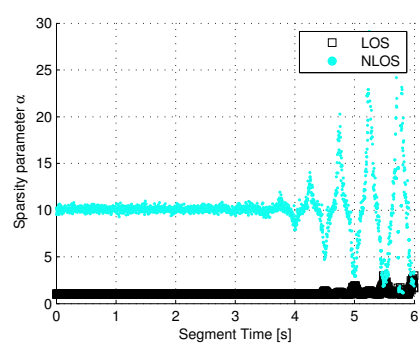

(b) Correlated components
Fig. 2: Sparsity parameter $\alpha$ for the different components.

the measured data, every second four super-frames were transmitted, leaving a $40 \mathrm{~ms}$ gap between each transmission.

\subsection{Simulated data}

In Fig. 1 estimated ranges for the assumption of uncorrelated and correlated components at an SNR of $15 \mathrm{dBm}$ are shown. The relative Doppler of of the multipath to the LOS is $2 \mathrm{~Hz}$, a realistic value for the environments under consideration. For better comparison to the standard method for range estimation, also a conventional time domain correlator is shown. From Fig. 1 it can be seen, that the correlator exhibits strong oscillations of up to $100 \mathrm{~m}$, caused by the superposition of paths with different Doppler frequencies. For both implementations, the number of components as well as their range can be estimated and tracked accurately, as long as their delay is still separated by more than a sample. However, once in the subsample region, for independent components, the algorithm is no longer able to separate the paths. This leads to the one detected component experiencing the same oscillations as for the standard correlator. For correlated components this behavior is not observed, the NLOS path is still detected.

This behavior can be best explained when looking at the sparsity parameter $\alpha$ in Fig. 2: For independent paths alpha diverges, when the paths relative delay decreases too strongly. This leads to the removal of the component. Using the assumption of correlated components however allows the paths still to be separated. Nevertheless, the estimation performance slightly suffers, leading to an incorrect estimation of both paths.

For a more quantitative assessment of the range errors, their histograms are plotted in Fig. 3. Herein large improvements over the standard correlator by the algorithm become apparent. While the application of a correlator results an root mean squared error of $38 \mathrm{~m}$, application of the algorithms leads to an reduction to $27 \mathrm{~m}$ for uncor-

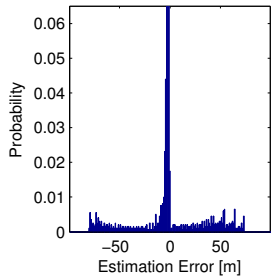

(a) Independent comp.

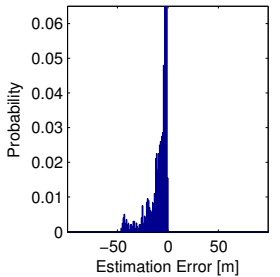

(b) Correlated comp.

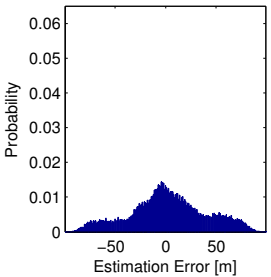

(c) Correlator
Fig. 3: Histogram for the range errors.

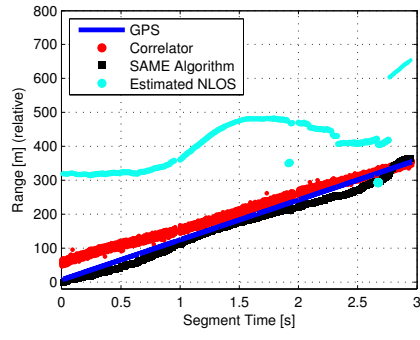

(a) Independent components

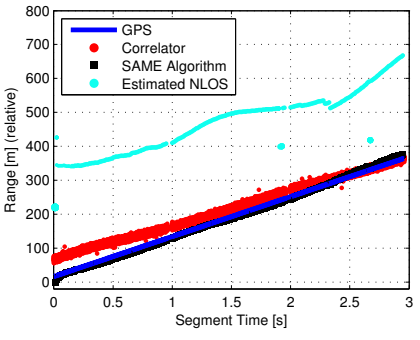

(b) Correlated components)
Fig. 4: Estimated LOS and NLOS components for measured data.

related, and $13 \mathrm{~m}$ for correlated components. Nevertheless, for the second implementation, reduction of the RMSE is traded in for an increase of the mean error.

\subsection{Measured data}

The measured data is taken from flight trials conducted in November 2012. For those measurements, a ground truth only exists for the line of sight path which is measured using the GPS positions ${ }^{2}$ for both the transmitting ground antenna and receiving aircraft antenna.

In Fig. 4 results from a three second segment of a low altitude flight at $2900 \mathrm{~m}$ is shown. It becomes obvious that the environment is influenced by multipath. A very prominent NLOS path is observed around $400 \mathrm{~m}$ relative the to the LOS. Another prominent multipath, detected and tracked by the algorithm at a range of around $2500 \mathrm{~m}$, is not shown for a better readability of the plots. Again, the algorithm leads to a reduction of the RMSE from $30 \mathrm{~m}$ for the normal correlator to $16 \mathrm{~m}$ and $6 \mathrm{~m}$ for the uncorrelated and correlated assumption.

\section{CONCLUSIONS}

In this work we discussed a novel algorithm for estimation and tracking of multipath components in multipath rich environments. Hereby, we compared two assumptions of independent and correlated multipath components. We have shown, that for both simulated and measured data, the proposed algorithms can lead to a significant improvement of the estimated range over the standard correlatorbased processing. Assuming correlations between the components generally allows tracking of close multipaths components; assuming independence the NLOS components the estimated models are sparser, i.e., the components are removed more often, leading to residual interference.

\footnotetext{
${ }^{2}$ An RTK (real time kinematics) GPS carrier phase position solution has an accuracy in the sub $10 \mathrm{~cm}$ region.
} 


\section{REFERENCES}

[1] M. Sajatovic, B. Haindl, C. Rihacek, M. Schnell, U. Epple, and S. Brandes, "L-DACS1 System Definition Proposal: Deliverable D3 - Design Specifications for L-DACS1 Prototype." 2009.

[2] D. Shutin, N. Schneckenburger, M. Walter, and M. Schnell, "LDACS1 Ranging Performance - An Analysis of Flight Measurement Results," in IEEEAIAA 32th Digital Avionics Systems Conference (DASC), (Syracuse, NY, USA), 2013.

[3] N. Schneckenburger, B. P. B. Elwischger, D. Shutin, M. Suess, B. Belabbas, and M. S. Circiu, "Positioning Results for LDACS1 Based Navigation with Measurement Data," in Proceedings of the ION GNSS + Meeting, The Institute of Navigation, (Nashville, TN, USA), 2013.

[4] D. Shutin, T. Buchgraber, S. R. Kulkarni, and H. V. Poor, "Fast Variational Sparse Bayesian Learning With Automatic Relevance Determination for Superimposed Signals," Transactions on Signal Processing, vol. 59, no. 12, pp. 6257-6261, 2011.

[5] H. Krim and M. Viberg, "Two decades of array signal processing research: the parametric approach," IEEE Signal Processing Mag., pp. 67-94, July 1996.

[6] M. Feder and E. Weinstein, "Parameter Estimation of Superimposed Signals Using the EM Algorithm," IEEE Trans. on Acoustics, Speech, and Sig. Proc., vol. 36, pp. 477-489, April 1988.

[7] B. Fleury, M. Tschudin, R. Heddergott, D. Dahlhaus, and K. I. Pedersen, "Channel parameter estimation in mobile radio environments using the SAGE algorithm," IEEE Journal on Sel. Areas in Comm., vol. 17, pp. 434-450, March 1999.

[8] E. Candes and M. Wakin, "An introduction to compressive sampling," IEEE Signal Process. Mag., vol. 25, pp. 21-30, Mar. 2008.

[9] D. Donoho, "Compressed sensing," IEEE Transactions on Information Theory, vol. 52, pp. 1289 -1306, april 2006.

[10] M. E. Tipping, "Sparse Bayesian learning and the relevance vector machine," The Journal of Machine Learning Research, 2001

[11] D. G. Tzikas, A. C. Likas, and N. P. Galatsanos, "The variational approximation for Bayesian inference," IEEE Signal Process. Mag., vol. 25, pp. 131-146, November 2008.

[12] D. Shutin and B. H. Fleury, "Sparse variational Bayesian SAGE algorithm with application to the estimation of multipath wireless channels," IEEE Trans. on Sig. Proces., vol. 59, pp. $3609-3623$, Aug. 2011.

[13] T. Jost, W. Wang, U.-c. Fiebig, and F. Pérez-fontán, "Detection and Tracking of Mobile Propagation Channel Paths," Transactions on Antennas and Propagation, vol. 60, no. 10, pp. 48754883, 2012.

[14] P. Stoica and Y. Selen, "Model-order selection: a review of information criterion rules," Signal Processing Magazine, IEEE, vol. 21 , no. 4, pp. 36-47, 2004

[15] B. Krach, P. Robertson, and R. Weigel, "An Efficient TwoFold Marginalized Bayesian Filter for Multipath Estimation in Satellite Navigation Receivers," EURASIP Journal on Advances in Signal Processing, vol. 2010, no. 1, p. 287215, 2010
[16] P. Closas, "A Bayesian approach to multipath mitigation in GNSS receivers," IEEE Journal of Selected Topics in Signal Processing, vol. 3, no. 4, pp. 695-706, 2009.

[17] D. Shutin and B. Fleury, "Sparse variational Bayesian SAGE algorithm with application to the estimation of multipath wireless channels," Signal Processing, IEEE Transactions on, vol. 59, no. 8, pp. 3609-3623, 2011.

[18] D. Shutin, W. Wang, and T. Jost, "Incremental Sparse Bayesian Learning for Parameter Estimation of Superimposed Signals," in 10th International Conference on Sampling Theory and Applications, no. 1, (Bremen, Germany), pp. 6-9, 2013.

[19] D. Shutin, T. Buchgraber, S. R. Kulkarni, and H. V. Poor, "Fast adaptive variational sparse Bayesian learning with automatic relevance determination," in IEEE Int. Conf. on Acoustics Speech and Sig. Proc., (Prague, Czech Republic), pp. 21802183, May 2011.

[20] C. M. Bishop, Pattern recognition and machine learning. Secaucus, NJ: Springer, 2006.

[21] S. M. Kay, Fundamentals of statistical signal processing, volume 1: estimation theory. Prentice Hall, 1993. 